\title{
About Boussinesq's turbulent viscosity hypothesis: historical remarks and a direct evaluation of its validity
}

\author{
François G. Schmitt \\ CNRS, FRE 2816 ELICO, Wimereux Marine Station, Université des Sciences et Technologies de Lille - Lille 1 \\ 28 av. Foch, 62930 Wimereux, France \\ francois.schmitt@univ-lille1.fr \\ Received $* * * * * ;$ accepted after revision +++++ \\ Presented by Patrick Huerre
}

\begin{abstract}
Boussinesq's hypothesis is at the heart of eddy viscosity models, which are used in many different fields to model turbulent flows. In its present time formulation, this hypothesis corresponds to an alignment between Reynolds stress and mean strain tensors. We begin with historical remarks on Boussinesq's results and recall that he introduced a local averaging twenty years before Reynolds, but using an approach that prevented him from discovering Reynolds' stress tensor. We then introduce an indicator that characterizes the validity of this hypothesis. For experimental and numerical databases, when the tensors are known, this can be used to directly estimate the validity of this hypothesis. We show, using several different databases, that this hypothesis is almost never verified. We address in conclusion the analogy with kinetic theory, and the reason why this analogy cannot be applied in general for turbulent flows.
\end{abstract}

\section{Résumé}

A propos de l'hypothèse de viscosité turbulente de Boussinesq : rappels historiques et évaluation directe. L'hypothèse de Boussinesq est au coeur des modèles de viscosité, utilisés dans un grand nombre de contextes pour modéliser des écoulements turbulents. Dans sa formulation moderne, cette hypothèse correspond à un alignement entre tenseur de contrainte de Reynolds et tenseur de déformation moyen. Nous rappelons le contexte historique de l'énoncé de cette hypothèse, en soulignant que Boussinesq avait introduit une moyenne locale vingt ans avant Reynolds, mais en effectuant une erreur qui l'a privé de la mise en évidence du tenseur de Reynolds. Nous introduisons ensuite un indicateur, compris entre 0 et 1 , indiquant le degré de validité de cette hypothèse. Pour des bases de données expérimentales et numériques, lorsque les différents tenseurs sont connus, ceci permet de tester directement, "a priori", cette hypothèse. Nous montrons ainsi, utilisant différentes bases de données d'écoulements turbulents, que l'hypothèse n'est presque jamais vérifiée. Nous discutons en conclusion de la théorie cinétique des gaz et de la raison pour laquelle cette analogie est discutable pour les écoulements turbulents.

Key words: Fluid Mechanics; Turbulence; Constitutive equation

Mots-clés : Mécanique des fluides; Turbulence; Equation constitutive

Preprint submitted to Elsevier Science

10 avril 2007 


\section{Version française abrégée}

Le "problème de la turbulence" touche un grand nombre de domaines, incluant l'ingénierie automobile, chimique, la combustion, l'aéronautique, la météorologie, l'océanologie, l'hydrologie, l'hydraulique fluviale, etc. Ces domaines sont à fort potentiel industriel et environnemental, et demandent souvent des réponses pratiques et quantitatives, faisant appel à des modèles. Parmi les différentes familles de modèles existant, beaucoup utilisent une moyenne de Reynolds, qui font intervenir les fluctuations instantannées à petites échelles via le tenseur de Reynolds. La modélisation intervient ici, et permet de fournir, via une hypothèse, une "fermeture" exprimant le tenseur de Reynolds en fonction de quantités moyennes. La fermeture la plus courante, fournissant le tenseur de Reynolds en fonction du champ de vitesse moyen, est celle qui est utilisée dans les modèles de viscosité turbulente, souvent appelée "hypothèse de Boussinesq". Il semble ici paradoxal de constater que la "moyenne de Reynolds" date d'une publication de 1895, tandis que l' "hypothèse de Boussinesq" permettant d'exprimer le tenseur de Reynolds est largement antérieure : la publication date de 1877, mais il s'agit d'un compte-rendu d'une séance de l'Académie des Sciences de 1872. Comment se fait-il que Boussinesq ait proposé en 1872 une fermeture pour une équation qui n'a été établie plus de 20 ans plus tard?

La réalité historique est un peu plus complexe que ce qui transparait dans les citations dans les ouvrages actuels sur la turbulence; nous pensons donc utile de revenir ici sur la publication originale de Boussinesq, disponible par exemple sur le site Gallica (http ://gallica.bnf.fr). Dans sa publication de 1877, Boussinesq effectue déjà une moyenne locale de l'écoulement, qu'il suppose stationnaire. Il considère les "composantes, suivant les axes, des actions exercées" à travers des plans. Il effectue alors un raisonnement qui est analogue à celui qui est utilisé en théorie cinétique, mais cette-fois ci pour les vitesses turbulentes : il obtient ainsi une équation de transport pour le champ moyen analogue à l'équation définie pour les valeurs instantanées. Boussinesq note bien que l'expression moyennée qu'il obtient obéit toujours aux équations de NavierStokes, mais qu'il faut remplacer le coefficient de viscosité par un nombre beaucoup plus grand, qu'il note $\epsilon$, qui n'est plus une constante et qui dépend "encore et surtout de l'intensité de l'agitation moyenne qui s'y trouve produite". Il donne ensuite pour ce coefficient une expression qui préfigure le modèle de longueur de mélange formalisé plus tard par Prandtl. En utilisant les termes actuels, on peut constater que Boussinesq a effectué à la fois (de façon simultanée) une moyenne des équations de Navier-Stokes, et l'introduction d'une modélisation tensorielle de type viscosité turbulente, en utilisant une analogie avec la théorie cinétique. Plus tard, Reynolds a été plus précis, utilisant le même cheminement, mais fournissant de façon explicite les équations moyennées; mais il ne mentionna pas les contributions de Boussinesq.

Dans une seconde partie, nous considérons l'hypothèse de Boussinesq formulée en utilisant les notations actuelles. Nous rappelons qu'elle correspond à un alignement entre deux tenseurs. Pour quantifier cette hypothèse, nous introduisons un indicateur qui est construit à partir d'un produit scalaire entre tenseurs. Cet indicateur est compris entre 0 et 1 , et vaut 1 lorsque l'hypothèse est vérifiée. Pour pouvoir estimer cet indicateur, il faut disposer de toutes les composantes du tenseur de Reynolds et du tenseur de déformation moyen. Dans une troisième partie, nous choisissons des bases de données turbulentes permettant d'estimer cet indicateur. Nous utilisons des données de simulation numérique directe (DNS), des données de simulation à grande échelle (LES), et des données expérimentales. Les résultats obtenus à partir de ces bases de données vont tous dans le même sens : l'hypothèse de Boussinesq, qui est à la base de nombreux modèles de viscosité turbulente, est rarement vérifiée. Le problème essentiel de cette fermeture est qu'elle repose sur une analogie avec la théorie cinétique, analogie dont nous rappelons la 
réfutation sur des bases théoriques dans la dernière partie. Nous rappelons que puisque la turbulence pleinement développée produit des statistiques fortement non gaussiennes, il est probable que les parcours individuels des élements de fluide fluctuent à un point tel que la prise en compte de leur effet collectif dans l'estimation de la contrainte moyenne, ne peut se résumer à un gradient local. Nous mentionnons dans ce contexte d'autres propositions qui ont été faites, telles que les modèles $K$ - $\epsilon$ non-linéaires, ou les modèles reposant sur d'autres équations constitutives non-linéaires possédant des effets de mémoire.

\section{Introduction}

The "turbulence problem" is present in many fields, including car industry, chemical engineering, combustion studies, aeronautics, meteorology, oceanography, hydrology, fluvial hydraulics, etc. These fields have a strong industrial and environmental potential, and often need practical and quantitative answers. As well known, turbulence is one of the last subjects of classical physics which is still not solved. This is why in engineering sciences, or in environmental studies, turbulence models are used $[1,2,3,4,5]$. Among the different existing models, many introduce Reynolds averaging [6]. In the original publication, Reynolds average correspond to average Navier-Stokes equations on boxes of intermediate size. In more recent formulations, the "Reynolds averages" that are estimated correspond to an ensemble average [7]. The objectives of these approaches is to discretize the modeled equations on a grid of adequate size, so that the processing time will not be too large. This is a way to decrease the number of degrees of freedom. Thus the idea is not to use a theory to provide an instantaneous turbulent solution, but to provide a mean numerical solution on a rough grid, using a model. The important point is the non-linearity of NavierStokes equations: the averaged equations are not closed and are expressed using small-scale instantaneous fluctuations, through the Reynolds tensor [6]. The introduction of a model is then necessary to close the equations, and provide, through an appropriate hypothesis, a "closure" expressing the Reynolds stress tensor using averaged quantities.

\section{Boussinesq and Reynolds: historical remarks}

The main closure, which provides the Reynolds stress tensor using the gradient of the mean velocity field, is used in turbulent viscosity models; it is often denoted "Boussinesq's hypothesis" (see some comments in [8], pp. 222-3). It seems here paradoxical to mention that for "Reynolds averages" a publication of 1895 is cited [6], whereas for "Boussinesq's hypothesis" which provides an expression for the Reynolds stress tensor, a publication of 1877 is cited, which is much earlier. This publication is in fact a report of a meeting of the French Academy of Science that was held during 1872. How is it possible that Joseph Boussinesq proposed in 1872 a closure for an equation that would be written more than 20 years later? Historical facts are somewhat more complex than what is usually mentioned in present time turbulence text books. We therefore think here that it is useful to go back to Boussinesq's original publication, which can be found in French at Gallica's web site (http://gallica.bnf.fr).

In his 1877 publication, Boussinesq performs already a local average of the flow, which is assumed stationary. He first considers temporal averages, done "during a rather short time $\tau$ " (p. 24). Soon after this, he assumes that velocity components are not correlated. He notes $u_{1}, v_{1}, w_{1}$ the components of the instantaneous velocity of an element of fluid, and $u=\overline{u_{1}}$ its temporal average. He then introduces the acceleration $u_{1}^{\prime}$ (Equation (2) page 28):

$$
u_{1}^{\prime}=\frac{d u_{1}}{d t}+u_{1} \frac{d u_{1}}{d x}+v_{1} \frac{d u_{1}}{d y}+w_{1} \frac{d u_{1}}{d z}
$$


We see here that he was not using the partial derivative notations. He then writes for the averaged acceleration $u^{\prime}=\overline{u_{1}^{\prime}}$ (Equation (3) page 29):

$$
u^{\prime}=\frac{d u}{d t}+u \frac{d u}{d x}+v \frac{d u}{d y}+w \frac{d u}{d z}
$$

corresponding to the assumption $\overline{u_{1} \partial_{x} v_{1}}=\overline{u_{1}} \partial_{x} \overline{v_{1}}$, and also to $\overline{u_{1} \partial_{x} u_{1}}=\overline{u_{1}} \partial_{x} \overline{u_{1}}$. He states further that there are some special situations (page 31): "when a group of molecules go up, we have at the same time $w_{1}>0$ et $\frac{d u_{1}}{d z}>0$, whereas we have $w_{1}<0$ et $\frac{d u_{1}}{d z}<0$ when the same group goes down. The product $w_{1} \frac{d u_{1}}{d z}$ is positive in both cases." He deduces from this that equation (2) is wrong in those cases, but he concludes "we see that this happens only in a relatively limited region and almost always negligible". In fact, the real situation is the opposite: here Boussinesq did not notice the apparition, through a temporal average of transport equations, of the tensor that will later be called Reynolds tensor. It is surprising in fact to discover that a few lines later, he implicitly introduces such a tensor when he considers the "components among the axes, of actions exerted" through planes of the fluid element. The average of the normal components of these actions are denoted $N_{1}, N_{2}$ and $N_{3}$, and of the tangential components $T_{1}$, $T_{2}$ and $T_{3}$. We recognise here the different components of the tensor $-\overline{u_{i} u_{j}}$, but this identification is not mentioned by Boussinesq. Through an approach which is similar to the one done in kinetic theory, but here for turbulent velocities, he performs a development in Taylor's series and obtains the different components of the "mean actions exerted through fixed planes" (page 42). Those are expressed as (equation (12) page 46):

$$
\begin{array}{ccc}
N_{1}=-p+2 \epsilon \frac{d u}{d x} \quad ; \quad & N_{2}=-p+2 \epsilon \frac{d v}{d y} ; & N_{3}=-p+2 \epsilon \frac{d w}{d z} \\
T_{1}=\epsilon\left(\frac{d v}{d z}+\frac{d w}{d y}\right) ; & T_{2}=\epsilon\left(\frac{d w}{d x}+\frac{d u}{d z}\right) ; & T_{3}=\epsilon\left(\frac{d u}{d y}+\frac{d v}{d x}\right)
\end{array}
$$

In this equation, $p$ is the pressure exerted on an element of fluid by the surrounding, given by (page 46):

$$
-\frac{1}{3}\left(N_{1}+N_{2}+N_{3}\right)=p-\frac{2}{3} \epsilon\left(\frac{d u}{d x}+\frac{d v}{d y}+\frac{d w}{d z}\right)
$$

We see here that for incompressible flows, and using usual present time notations, we have $p=\frac{2}{3} K$, where $K$ is the kinetic energy, written as $2 K=-\left(N_{1}+N_{2}+N_{3}\right)$. The equation (3) given by Boussinesq is in fact a tensorial closure providing stresses on the form:

$$
-\frac{2}{3} K \delta_{i j}+\epsilon\left(\frac{d u_{i}}{d x_{j}}+\frac{d u_{j}}{d x_{i}}\right)
$$

Boussinesq remarks that the average expression he obtains still obeys Navier-Stokes equations, where the viscosity coefficient is replaced by a number which is much larger, denoted $\epsilon$. This is no more a constant, and depends "mainly on the mean agitation which is produced". An expression is proposed by Boussinesq for $\epsilon$ :

$$
\epsilon=\rho g \Lambda h u_{0}
$$

where $\rho g$ is the weight of a volume unity, $\Lambda$ is a scalar, slowly varying with $h$ and $u_{0}$, which are respectively a characteristic length and velocity. This relation for $\epsilon$ which is now denoted as "turbulent viscosity", or "effective viscosity", is already a mixing length formulation, as developed later by Prandtl [10].

We thus see that, despite an error in the beginning concerning the correlations of velocity components, "Boussinesq's hypothesis" was originally explicitly mentioned as a tensorial relation, with a justification 
linked to mixing length arguments. Using present day vocabulary, we see that Boussinesq performed simultaneously an average of Navier-Stokes equations and a tensorial closure of the eddy-viscosity type, using an analogy to kinetic theory.

Boussinesq thought that he solved the turbulence problem; he did not realize his mistake in the average of Navier-Stokes equations, neither did he notice that he was assuming a strong hypothesis when he performed his analogy with kinetic theory. In the same volume, the 1877 contribution of Boussinesq is introduced by Saint Venant (a 22 pp. introduction) [11]. The latter writes that Boussinesq solved a "true enigma". Much later, in his posthumous speech on Boussinesq's achievements, the President of the French Academy of Sciences Emile Picard mention his results as if they were exact results: "It is a remarkable result due to Boussinesq, that Navier's equations are still valid, but introducing, instead of real molecular velocities, their local averages." ([12], p. 78). On the other hand, it is surprising to notice that Boussinesq does not cite the 1895 work of Reynolds in his 1897 book. In this paper, Reynolds undertook the same averaging approach, but with a more rigourous line, introducing the stress tensor. There is a citation default in both cases, since Reynolds did not cite the 1877 results of Boussinesq on local averages.

Let us note here that neither Boussinesq nor Reynolds seem to have used the word "turbulence" in their publications: Boussinesq $[13,9,16]$ used "tumultuous movements", "eddy agitations", "movements theory", "liquid eddy theory" (in French, "mouvements tumultueux", "agitation tourbillonnaire", "théorie des remous", "théorie des tourbillons liquides", "force vive"), whereas Reynolds [14,6] used "sinuous paths", "sinuous motion", "irregular eddies", "sinuous or relative disturbance". The word turbulence seems to have been introduced in the field of fluid mechanics for the first time by William Thomson (later Lord Kelvin) in 1887 [15]. This word was not adopted by Boussinesq or Reynolds in their works posterior to 1887 (see the title of Boussinesq, 1897: Theory of tumultuous and whirling liquid flows [16]); none of them did cite Kelvin in their 1890s publications $[6,16]$. The word turbulence was adopted in the field only later, in the beginning of the XXth century, in particular through the works of Prandtl.

\section{Direct test of Boussinesq's hypothesis}

We come back here to turbulent viscosity models. Reynolds averaging of Navier Stokes equations is written using the Reynolds stress tensor $\left\langle u_{i} u_{j}\right\rangle$, where $\left(u_{i}\right)$ is the fluctuating velocity, $\left(U_{i}\right)$ the mean velocity, and $\langle$. $>$ represents ensemble averaging. To achieve closure of the mean equations, it is necessary to express the Reynolds tensor from the mean velocity, and other mean quantities. Among the latter is the mean kinetic energy $K=\frac{1}{2}<u_{i} u_{i}>$. We introduce also the traceless stress tensor, also sometimes called anisotropic stress tensor: $\mathbf{R}=<u_{i} u_{j}>-\frac{2}{3} K \mathbf{I}$ where bold notation is used for tensors, and $\mathbf{I}$ is the unit tensor. We also introduce the mean velocity gradient tensor $\mathbf{A}=\partial U_{i} / \partial x_{j}$ and its symmetric part, giving the mean strain tensor:

$$
\mathbf{S}=\frac{1}{2}\left(\frac{\partial U_{j}}{\partial x_{i}}+\frac{\partial U_{i}}{\partial x_{j}}\right)
$$

whose trace vanishes for incompressible flows. Boussinesq's hypothesis corresponds then to a closure hypothesis with the following linear constitutive equation:

$$
\mathbf{R}=-2 \nu_{T} \mathbf{S}
$$

where $\nu_{T}$ is a scalar coefficient called turbulent viscosity (sometimes called "effective viscosity"). This equation is a linear relation between stress and strain tensors, and is analogous to the linear constitutive equation for Newtonian flows: $\mathbf{R}_{\nu}=-2 \nu \mathbf{S}$, where $\mathbf{R}_{\nu}$ is the viscous stress tensor and $\nu$ the viscosity. 
In Equation (8), the eddy-viscosity is written for the classical $K-\epsilon$ model [17] using two independent turbulent quantities such as $K$ and the dissipation $\epsilon: \nu_{T}=C_{\mu} K^{2} / \epsilon$, where $C_{\mu}$ is a non-dimensional quantity (in some recent models it is no more constant). Closure is achieved with transport equations for $K$ and $\epsilon$. Here we do not consider transport equations.

The main hypothesis of equation (8) is here the fact that the 2 tensors are proportional. This proportionality can be easily tested when the two tensors $\mathbf{R}$ and $\mathbf{S}$ are known. For this, we introduce an inner product (or "contracted product") between matrices that writes $\mathbf{A}: \mathbf{B}=\left\{\mathbf{A}^{t} \mathbf{B}\right\}=A_{i j} B_{i j}$, where $\{$.$\} is the trace. The associated norm is the following: \|\mathbf{A}\|^{2}=\mathbf{A}: \mathbf{A}$. For symmetric tensors, one has $\mathbf{A}: \mathbf{B}=\{\mathbf{A B}\}$ and $\|\mathbf{A}\|^{2}=\left\{\mathbf{A}^{2}\right\}$. This can be used to introduce an indicator $\rho_{R S}$ which is defined through an inner product between tensors [18]:

$$
\rho_{R S}=\frac{|\mathbf{R}: \mathbf{S}|}{\|\mathbf{R}\| \mid\|\mathbf{S}\|}
$$

which is analogous to the cosine of the angle between vectors. The ratio $\rho_{R S}$ is thus a number between 0 and 1 , which characterizes the validity of Boussinesqs hypothesis: it is 1 when this hypothesis is valid, and the less it is valid, the more this number is close to 0 (this corresponds to "perpendicular" tensors). Pushing further the analogy with an angle between vectors, one can consider that alignment is approximatively verified for angles smaller than $\pi / 6$, corresponding to a value of $\rho_{R S}$ larger than 0.86 . This indicator is thus a direct indicator of the validity of the basic hypothesis of all linear eddy viscosity models. To estimate this number, one needs to have access to all the components of the Reynolds stress and mean strain tensors in order to estimate the invariants $\{\mathbf{R S}\},\left\{\mathbf{R}^{2}\right\}$ and $\left\{\mathbf{S}^{2}\right\}$. The stress tensor is often completely or partially given in turbulent databases through turbulence intensity components and the various shear stress terms; the strain tensor is less often available, since it involves derivatives in all directions, whose estimation requires the data base to be provided on a fine enough grid. Nevertheless, except for complex 3D flows, available databases correspond to simplified geometries possessing symmetries, so that many components of the Reynolds stress and mean strain tensors vanish; this is the case for most of the databases tested here, corresponding to 2D mean flows and an axisymmetric jet flow.

This indicator is applied below to numerical and experimental databases.

\section{Application of the indicator to numerical and experimental databases}

\subsection{Direct numerical simulation data of simple flows}

The DNS data analyzed here correspond to simple shear flows possessing only one non-zero velocity gradient Some of these data bases have been studied in details elsewhere [19]. The first database (denoted $\mathrm{CO}$ in the following) corresponds to a turbulent plane Couette flow at a Reynolds number $R e=1300$ with a friction Reynolds number $R e_{\tau}=82$ [20]. The second database (denoted CF87 in the following) corresponds to a turbulent channel flow at a Reynolds number $R e=3250$ corresponding to a friction Reynolds number of $R e_{\tau}=180$ [21], and the third (denoted CF99) is the same flow at larger Reynolds number: $R e=10^{4}$ corresponding to a friction Reynolds number of $R e_{\tau}=590$ [22]. The fourth database (denoted BL in the following) is a turbulent boundary layer on a flat plate, with zero pressure gradient, with a Reynolds number of $R e=210^{5}$ or based on the momentum thickness $\theta, R_{\theta}=1410$ [23]. The last DNS database corresponds to an annular pipe flow (denoted AP) with a bulk Reynolds number of $R e=2800$ (based on bulk velocity and $\delta$, the half-width of the annular gap) or friction Reynolds number $R e_{\tau}=180$ [24]. The inner diameter is denoted $R_{i}$ and the outer is $R_{o}=2 R_{i}$. The adimensional radial distance $r=\left(d-R_{i}\right) / \delta$ is then a number between 0 and 2 . 


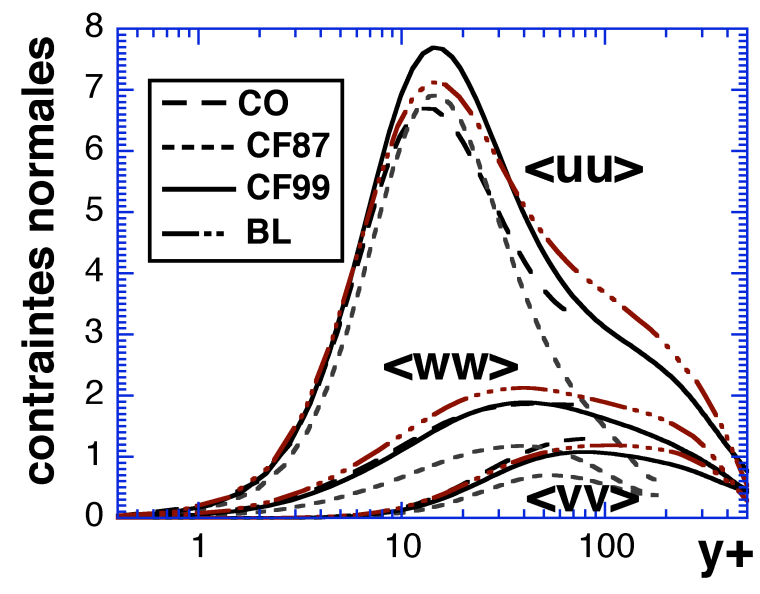

Figure 1. Normal stresses for DNS databases.

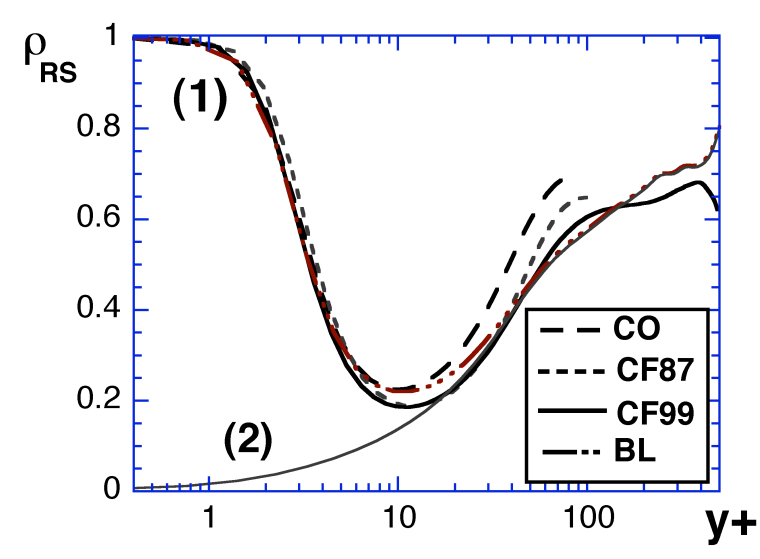

Figure 2. Plot of $\rho_{R S}\left(y^{+}\right)$for DNS databases: (1) total stress (turbulent+viscous stress) and (2) turbulent stress only.

The DNS test cases chosen correspond to a panel of Reynolds numbers going from $10^{3}$ to $10^{5}$, with friction Reynolds numbers from 82 to 1410. The friction Reynolds numbers above are defined using the friction velocity $u_{\tau}=\sqrt{\tau_{w} / \rho}$, where $\tau_{w}$ is the modulus of the wall shear stress. In the following, most quantities are non-dimensionalized, using $u_{\tau}$ or $u_{\tau}^{2}$; the distance to the wall is expressed as usual in wall units (also a local Reynolds number) $y^{+}=y u_{\tau} / \nu$, (except for the AP database where natural coordinate is kept in order to visualise in the same graph the behaviour near the two walls). For these shear flows, only $1 \mathrm{D}$ profiles are necessary. All the profiles of the Reynolds stress tensors are usually provided. Furthermore, the only non-zero gradient is $a=U^{\prime}(y)$ whose knowledge provides the mean strain tensor:

$$
\mathbf{S}=\frac{a}{2}\left(\begin{array}{lll}
0 & 1 & 0 \\
1 & 0 & 0 \\
0 & 0 & 0
\end{array}\right)
$$

Figure 1 represents the normalized normal stresses for the data sets, showing that the anisotropy of the stresses is pronounced. It is well-known (see e.g. $[25,26]$ ) that since normal stresses are not identical (corresponding to anisotropy), Boussinesq's hypothesis cannot be valid. Indeed, the anisotropic stress tensor is here:

$$
\mathbf{R}=\left(\begin{array}{ccc}
-\frac{2}{3} K+\left\langle u^{2}\right\rangle & \langle u v\rangle & 0 \\
\langle u v\rangle & -\frac{2}{3} K+\left\langle v^{2}\right\rangle & 0 \\
0 & 0 & -\frac{2}{3} K+\left\langle w^{2}\right\rangle
\end{array}\right)
$$

and for the latter to be proportional to $\mathbf{S}$, it would be necessary that diagonal terms vanish.

Many authors have mentioned that Boussinesq's hypothesis is not correct for simple shear flows; our contribution in this context is here to use the representation of the indicator $\rho_{R S}\left(y^{+}\right)$to quantify the degree of validity of Boussinesq's hypothesis (Figure 2). In this figure, we have represented the total stress and the turbulent stress alone. Very close to the wall, the viscous stress is dominant as expected, and the total stress is aligned to the strain tensor. Moreover, this figure directly shows that the viscous term has an influence on the total stress until a distance of $y^{+}=20$, but the alignment is bad for for $y^{+}>2$. 


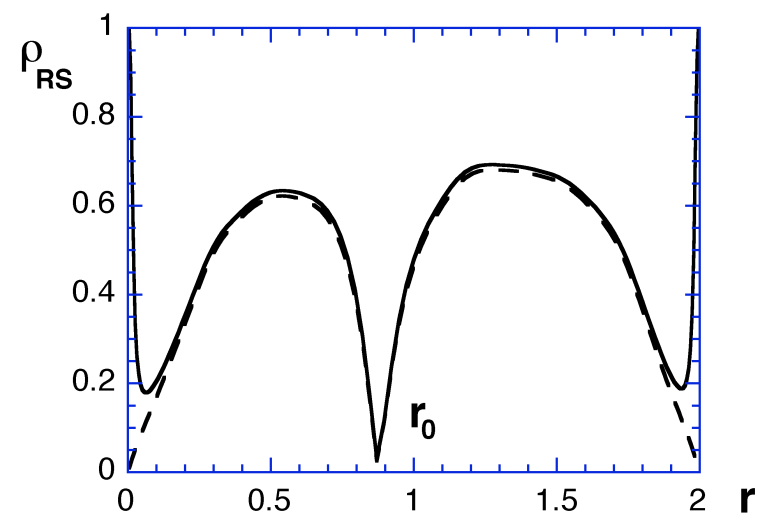

Figure 3. Plot of $\rho_{R S}(r)$ for the DNS database AP: total stress (continuous line) and turbulent stress alone (dotted line).

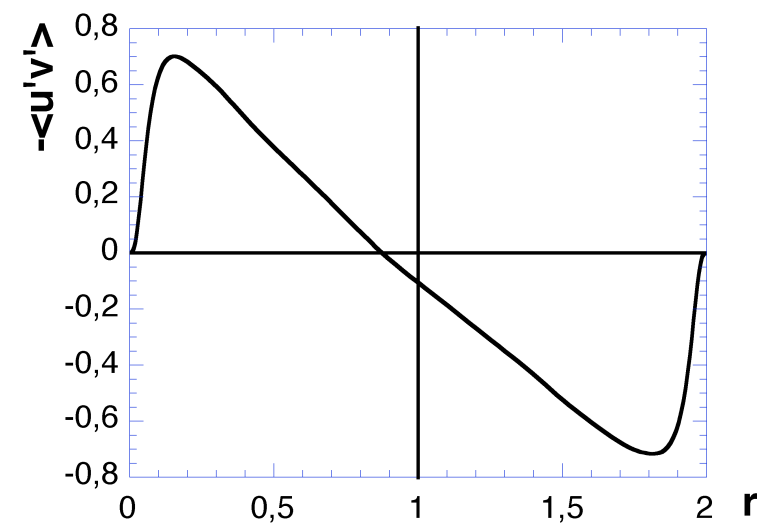

Figure 4. Plot of the shear stress for the AP DNS database.

Globally, for the total stress, the alignment, corresponding to the validity of Boussinesq's hypothesis, is bad for $3<y^{+}<70$, (corresponding roughly to the buffer layer). There is a local minimum at about the same position for all data bases, $y^{+}=10.5 \pm 0.5$. The different curves superpose rather well: the data bases have approximatively the same behaviour, indicating a possible universality in the failure of this linear constitutive relation near the walls.

The annular pipe flow presents some interesting non-symmetry effects (between inner and outer cylinders). For this database $\rho_{R S}$ is shown in real instead of wall units in Figure 3. For this flow, this ratio has an interesting shape. Boussinesqs hypothesis is never valid (except very close to the walls for the total stress), but it is the worse at the position $r_{0}=0.87$ corresponding to an asymmetric center position, which is closer to the inner cylinder than the outer one. As shown in Figure 4, this position corresponds to the annulation of the mean velocity gradient tensor. The shear stress also vanishes at this position, whereas normal stresses are not isotropic, so that $\mathbf{R}$ is not vanishing, but is diagonal, whereas the mean strain tensor is vanishing:

$$
\mathbf{R}\left(r_{0}\right)=\left(\begin{array}{ccc}
-0.19 & 0 & 0 \\
0 & 0.10 & 0 \\
0 & 0 & 0.09
\end{array}\right) ; \mathbf{S}\left(r_{0}\right)=0
$$

The different turbulent values at this position correspond then to the non-validity of Boussinesq's hypothesis and also of any polynomial non-linear constitutive equation such as used in non-linear $K-\epsilon$ models, as discussed below.

\subsection{LES data}

The LES (Large Eddy Simulation) data considered here correspond to the flow over a square cylinder, with a Reynolds number of $R e=22000$. This geometry was used as a test case in a workshop on LES of flows past bluff bodies held in 1995 in Germany (see a report in [27]). The data analyzed here were provided by the university of Surrey, UK. The mesh size is $257 \times 241 \times 64$; the mean flow is $2 \mathrm{D}$ and averages are taken over time (56 different time sections) and the spanwise dimension (64 values). This provides $64 \times 56=3584$ samples which were used to compute mean quantities and second moments 


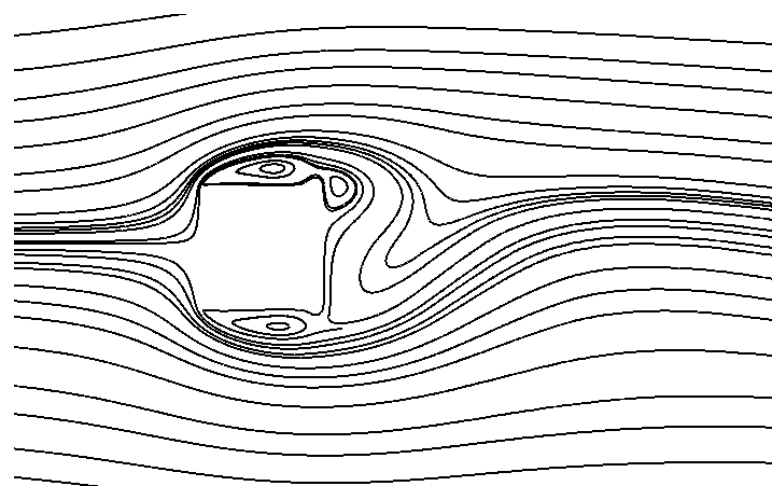

Figure 5. Streamlines of the flow past a square cylinder estimated from LES data.

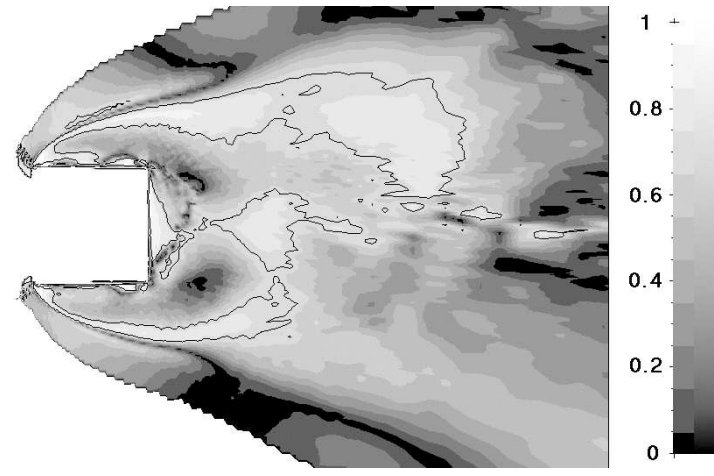

Figure 6. A map of $\rho_{R S}$ for the LES database. The inflow region is not plotted. The isoline corresponds to an "angle" of $\pi / 4$. Only the region inside this isoline corresponds approximatively to Boussinesq's hypothesis.

such as all components of the Reynolds stress tensor. Figure 5 shows the streamlines of the mean flow. Gradients were estimated using a second order finite difference scheme, giving access to all components of the mean strain and vorticity tensors.

This database has been used elsewhere to check the kinetic energy transport equation [28]. Figure 6 represents $\rho_{R S}$ estimated for the whole region: light regions indicate validity of Boussinesq's hypothesis (value close to 1 ). An isoline represents the value $\rho=\cos \pi / 4 \approx 0.71$, corresponding to a roughly valid linear hypothesis. The region inside this isoline is not of wide extension. Since the tensors are very small in the inflow region, the alignment ratio is not plotted to avoid unnecessary scatter. Contrary to previous examples, this corresponds to a complex flow, with mixing and recirculation regions. A LES database has been chosen since DNS is not yet possible for such flow. The result shown here confirms previous results obtained for simple shear flows.

\subsection{Experimental data}

We consider also here an experimental database, corresponding to a double annular turbulent jet flow, generated by a confined double annular burner in cold conditions. Two dimensional LDV measurements have been performed on 5515 grid points close to the nozzle exit, where the flow is characterized by vortices, recirculation, high mixing rate. At each measurement position, statistics have been computed on 3000 to 16000 particles. The resulting mean flow field is axisymmetric within 2 p.cent. The nonzero components of the mean velocity gradient tensor have been computed on the fine grid, so that the above procedure can be applied to test Boussinesq's hypothesis with the indicator $\rho_{R S}$ computed at each grid position. The data base is available online (http://stro9.vub.ac.be/expdata); a complete description is given in [29]. Figure 7 below shows the streamlines of the flow analyzed. Figure 8 shows a map of $\rho_{R S}$. Validity of Boussinesq's hypothesis are represented by white regions, which are of relatively limited extension. Despite important noise due to the experimental nature of the database, we can say that Boussinesq's hypothesis is not validated for such complex flow. 


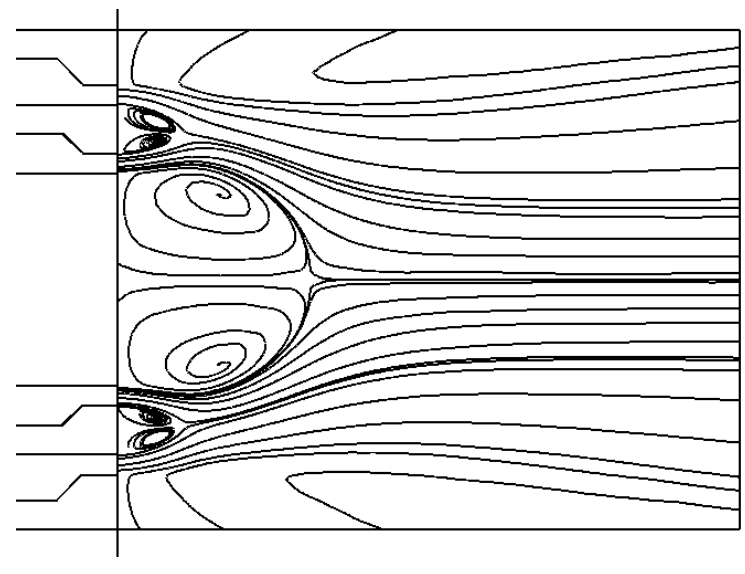

Figure 7. Streamlines of the double annular jet flow estimated from experimental data, showing recirculation and mixing regions.

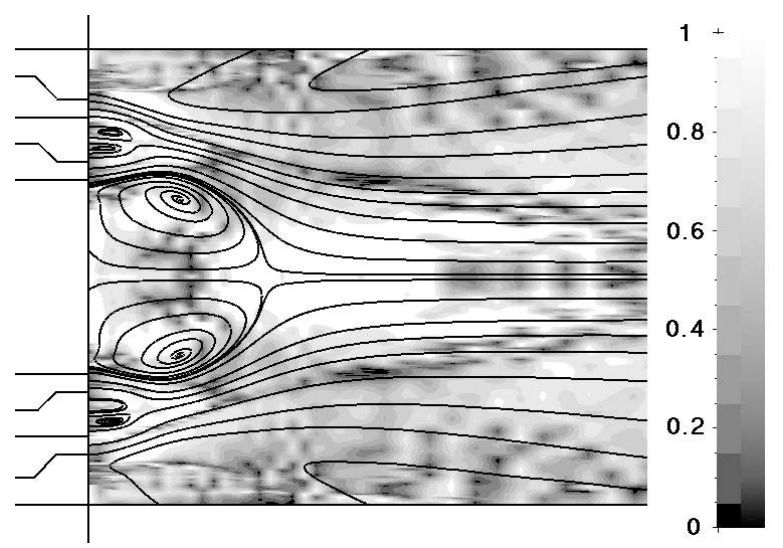

Figure 8. A map of $\rho_{R S}$ for the experimental double annular jet data. Only white regions correspond to validity of Boussinesq's hypothesis.

\section{Discussion and conclusion}

The results obtained using numerical (DNS or LES) or experimental data are very consistent in pointing the non-validity of Boussinesq's hypothesis, which is at the heart of many turbulent viscosity models. However, viscosity turbulence models such as $K-\epsilon$ model are widely used for many applications, and seem to provide satisfactory predictions. This may be seen as a contradiction. We must note in fact that these models predict rather closely only simple flows, and only as far as mean fields are concerned (mean velocities, streamflows). For example, in simple shear flows the shear stress is dominant in mean transport equations, so that the failure of the linear constitutive equation does not have important consequences for the final result. But predictions for second order moments (Reynolds stresses, kinetic energy, dissipation) are not satisfactotry, even for simple flows. And for complex flows, having high mixing rates, recirculation regions, stagnation lines, the predictions of these models can be qualitatively wrong, even for first moments (see [3] for more comments). The inaccuracies come from the transport equations and the linear constitutive equation.

The main limitation of this linear closure is the fact that it rests on an analogy with kinetic theory, analogy that can be criticised on theorical grounds, as discussed below (see also discussion in $[1,3,5]$ ).

\subsection{Kinetic theory and scale separation}

Eddy-viscosity turbulence models are the subject of thousands of papers, yet only a very small number of them discuss the basic theoretical weaknesses of Boussinesq and the gradient diffusion hypothesis when applied to turbulent flows (see e.g. [33,34,35,36,37,38]). As already implicitly assumed by Boussinesq, the basis of such modelling is an analogy with kinetic theory, where the viscous stress is expressed using the gradient of the mean field (see also the comments in [3] ou [5]). The simple situation where molecules have a mean velocity of the form $(U(y), 0,0)$ is often taken as an illustrative example: the gradients are in fact an approximation of the finite difference quantity $U\left(y+\ell_{m}\right)-U\left(y-\ell_{m}\right)$, where $\ell_{m}$ is the mean-free path of molecules. Since this distance is very small compared to the scale of variation of $U$, the following approximation is clearly justified:

$$
U\left(y+\ell_{m}\right)-U\left(y-\ell_{m}\right)=2 \ell_{m} \frac{\partial U}{\partial y}
$$


For turbulent flows the same construction is implicitely assumed, taking for $\ell_{m}$ the mean free path of fluid elements, i.e. the turbulent mixing length introduced by Prandtl. For this, the hypotheses below must be realised:

- each element of fluid possesses a turbulent lagrangian free path $\ell$, which is a random variable whose distribution is peaked around a mean value $\ell_{m}$, the turbulent mixing length;

- the turbulent mixing length is small compared to the scale of variation of mean quantities.

The free path of an element of fluid in the Lagrangian framework can be defined with the Lagrangian autocorrelation function. Indeed, for each Lagrangian trajectory a characteristic time (the integral of the autocorrelation function) can be estimated, providing a characteristic length, which is the mixing length associated to the trajectory. But the second hypothesis above is less justified: since fully turbulent flows possess high non-gaussian variability, it is likely that individual free paths take a whole range of values so that their collective effect in the computation of the stress cannot resume in just one difference such as given in (13). Furthermore, their mean is interpreted as the turbulent mixing length, which is generally not small compared to the scale of variation of the mean velocity, so that the separation of scales does not hold in general for turbulent flows [1].

\subsection{Nonlinear developments of Boussinesq's hypothesis}

Since Pope [30], it is recognized that invariant theory (and especially results obtained in the fifties by Rivlin and Spencer, see [31]) can be used in the framework of turbulence modeling to represent the stress tensor as a development into a tensor basis composed of no more than 10 basis tensors. This provides the following general development:

$$
\mathbf{R}=\sum_{i=1}^{10} a_{i} \mathbf{T}_{i}
$$

where the coefficients $a_{i}$ are scalar invariants of the flow, which are scalar fields having values independent of the system of reference, corresponding to traces of different tensor products [31]. In nonlinear eddyviscosity turbulence models (see e.g. [32,25,26]), a nonlinear constitutive relation such as (14) is used, with a tensorial basis $\mathbf{T}_{i}$ that can be expressed on the form of products of the mean strain tensor $\mathbf{S}$ and of the mean vorticity tensor W. Most of these models are polynomials, of degree 2 or 3 . These models generalise models of the $K-\epsilon$ family and are often called nonlinear $K-\epsilon$ models [3]. These nonlinear generalizations of Boussinesq's hypothesis help to overcome the clear limitations of the linear framework, but in a too superficial way, since they still provide closure using gradients of mean quantities; using DNS data of an annular pipe flow, we have shown above that, at a near central position, a nonlinear polynomial constitutive equation is not adequate since the strain and vorticity tensors vanish, whereas the anisotropic stress does not. This example shows that such non-linear constitutive equation cannot represent a full and consistent answer to the closure problem. The development of a new approach, more deeply connected to the multiscale and long-range correlated nature of fully developed turbulence seems desirable, providing in particular a turbulent constitutive relation of a new type. An interesting direction for further research is provided by non-local models, that take into account the history of turbulence: several models have already been proposed $[34,36,38,35,39]$, and an explicit expression for the kernel of the non-local model has recently been proposed and tested on simple flows [40]. Direct analysis of DNS data, using a Lagrangian approach to follow elements of fluid, could also be a good way to better understand the mechanism inside the formation of the turbulent stress [37,41], and improve the recent proposal of Hamba [40].

Turbulence models have important engineering applications, and thus in this framework, having models whose predictions are only partly in agreement with reality is better than having no agreement at all. 
To this aim, available turbulence models possess several tuning parameters, that are determined using $a$ posteriori validation. This type of validation is often restricted to mean profiles, and the comparison is qualitative. Depending on the type of application considered, such qualitative prediction may be enough; it is nevertheless certainly better to be able to provide good quantitative predictions for second and higher moments. Indeed, depending on the application foreseen, fluxes and even extreme and rare events can be important (e.g. for building structures able to resist strong winds); furthermore, fully developed turbulence develops intermittent fluctuations and contains long-range correlations (see reviews in $[8,3]$ ). Such effects are not considered in classical eddy-viscosity models, and thus are not predicted. It is in this respect that direct a priori tests are useful, in order to better assess the source of the weaknesses of available models, to be able to cure these weaknesses and develop new turbulence models.

\section{Acknowledgements}

We thank Pr. P. Voke for providing us the LES database, Dr. M. Quadrio for sending us his DNS data, C. Hirsch, G. Mompean, B. Merci for discussions, and reviewers for interesting suggestions.

\section{References}

[1] H. Tennekes, J. L. Lumley, A first course in turbulence, MIT Press, Cambridge, 1972.

[2] D. C. Wilcox, Turbulent modeling for CFD, second ed., DCW Industries, La Canada, 1998.

[3] S. B. Pope, Turbulent flows, Cambridge University Press, Cambridge, 2000.

[4] J. Mathieu, J. Scott, An introduction to turbulent flow, Cambridge University Press, Cambridge, 2000.

[5] P. S. Bernard, J. M. Wallace, Turbulent flow; Analysis, Measurement, and Prediction, John Wiley and sons, Hoboken, 2002 .

[6] O. Reynolds, On the dynamical theory of incompressible viscous fluids and the determination of the criterion, Phil. Trans. R. Soc. London A 186 (1895) 123-164.

[7] Osborne Reynolds Centenary volume, Proc. R. Soc. London A 451 (1995) 1-318.

[8] U. Frisch, Turbulence; the legacy of A. N. Kolmogorov, Cambridge University Press, Cambridge, 1995.

[9] J. Boussinesq, Essai sur la théorie des eaux courantes, Mémoires présentés par divers savants à l'Académie des Sciences XXIII, 1 (1877) 1-680.

[10] L. Prandtl, Bericht ber Untersuchungen zur ausgebildeten Turbulenz, Z. Angew. Math. Mech., 5 (1925) 136-9.

[11] Saint-Venant, Rapport sur un mémoire de M. Boussinesq, Mémoires présentés par divers savants à l'Académie des Sciences XXIII, 1 (1877) I-XXII.

[12] E. Picard, Discours et notices, Gauthier-Villars, Paris, 1936.

[13] J. Boussinesq, Mémoire sur l'influence des frottements dans les mouvements réguliers des fluides, J. Math. Pures Appl. sér. II, 13 (1868) 377-423.

[14] O. Reynolds, An experimental investigation of the circumstances which determine whether the motion of water shall be direct or sinuous, and of the law of resistance in parallel channels, Phil. Trans. R. Soc. London A 174 (1883) 935-982.

[15] Lord Kelvin (W. Thomson), On the propagation of laminar motion through a turbulently moving inviscid liquid, Phil. Mag. 24 (1887) 342-353.

[16] J. Boussinesq, Théorie de l'écoulement tourbillonnant et tumultueux des liquides, Gauthier-Villars et fils, Paris, 1897.

[17] B. E. Launder, R. Spalding, The numerical computation of turbulent flows, Computer Meth. Applied Mech. Eng. 3 (1974) 269-289.

[18] F. G. Schmitt, C. Hirsch, Experimental study of the constitutive equation for an axisymetric complex turbulent flow, Zeit. ang. Math. Mech. 80 (2000) 815-825. 
[19] F. G. Schmitt, Direct test of a nonlinear constitutive equation for simple turbulent shear flows using DNS data, Comm. Nonlinear. Sci. Num. Sim. (2007) in press.

[20] K. H. Bech et al., An investigation of turbulent plane Couette flow at low Reynolds numbers, J. Fluid Mech. 286 (1995) 291-325

[21] J. Kim, P. Moin, R. Moser, Turbulence statistics in fully developed channel flow at low Reynolds number, J. Fluid Mech. 177 (1987) 133-166

[22] R. D. Moser, J. Kim, N.N. Mansour, Direct numerical simulation of turbulent channel flow up to Re $\tau=590$, Physics of Fluids 11 (1999) 943-945.

[23] P. Spalart, Direct simulation of a turbulent boundary layer up to $R t=1410$, J. Fluid Mech. 187 (1988) 61-98.

[24] M. Quadrio, P. Luchini, Direct numerical simulation of the turbulent flow in a pipe with annular cross-section, Eur. J. Mech. Fluid Ser. 21 (2002) 413-27.

[25] S. Nisizima, A. Yoshizawa, Turbulent channel and Couette flows using a anisotropic $k$ - $\epsilon$ model, AIAA J. 25 (1986) 414-420.

[26] C. G. Speziale, On nonlinear $k-\ell$ and $k-\epsilon$ models of turbulence, J. Fluid Mech. 178 (1987) 458.

[27] W. Rodi, J. H. Ferziger, M. Breuer, M. Pourquié, Status of Large Eddy Simulation: Results of a Workshop, Tr. ASME: J. Fluid Eng. 119 (1997) 248-262.

[28] F. G. Schmitt, B. Merci, E. Dick, C. Hirsch, Direct investigation of the K-transport equation for a complex turbulent flow, J. Turbulence 3 (2003) 021.

[29] F. Schmitt, B. K. Hazarika, C. Hirsch, LDV measurements of the flow field in the nozzle region of a confined double annular burner, Tr. ASME: J. Fluid Eng. 123 (2001) 228-236.

[30] S. P. Pope, A more general effective-viscosity hypothesis, J. Fluid Mech. 72 (1975) 331-340.

[31] A. J. Spencer, Theory of invariants, in A. C. Eringen (Ed.), Continuum Physics, Academic Press, New York, 1971 , vol. 1, pp. 239-353.

[32] A. Yoshizawa, Statistical analysis of the deviation of the Reynolds stress from its eddy-viscosity representation, Physics of Fluids 27 (1984) 1377-1387.

[33] J. L. Lumley, Toward a turbulent constitutive relation, J. Fluid Mech. 41 (1970) 413-434.

[34] S. Corrsin, Limitations of gradient transport models in random walks and turbulence, Adv. Geophys. 18A (1974) 25-60.

[35] P. W. Egolf, Difference-quotient model: a generalization of Prandtls mixing-length theory, Physical Review E 49 (1994) 1260-1268.

[36] J. O. Hinze, R. E. Sonnenberg, P. J. H. Builtjes, Memory effect in a turbulent boundary-layer flow due to a relatively strong axial variation of the mean-velocity gradient, Applied Scientific Research 29 (1974) 1-13.

[37] P. Bernard, R. Handler, Reynolds stress and the physics of turbulent momentum transport, J. Fluid Mech. 220 (1990) 99-124.

[38] S. Tavoularis, S. Corrsin S., Experiments in nearly homogeneous turbulent shear flow with a uniform mean temperature gradient, part I, J. Fluid Mech. 104 (1981) 311-347.

[39] Y. N. Huang, On modelling the Reynolds stress in the context of continuum mechanics, Comm. Nonlinear. Sci. Num. Sim. 9 (2004) 543-559.

[40] F. Hamba, Nonlocal analysis of the Reynolds stress in turbulent shear flow, Phys. Fluids 17 (2005) 115102.

[41] R. A. Handler, P. S. Bernard, On the role of accerelating fluid particles in the generation of Reynolds stress, Phys. Fluids A 4 (1992) 1317-1319. 